\title{
Use of Langmuir probes in non-Maxwellian space plasmas
}

\author{
Walter R. Hoegy \\ NASA/Laboratory for Atmospheres Goddard Space Flight Center, Greenbelt, Maryland 20771 \\ Larry $\mathrm{H}$. Brace \\ Space Physics Research Lab, The University of Michigan, Ann Arbor, Michigan 48109
}

(Received 19 November 1998; accepted for publication 1 April 1999)

\begin{abstract}
Disturbance of the Maxwellian plasma may occur in the vicinity of a spacecraft due to photoemission, interactions between the spacecraft and thermospheric gases, or electron emissions from other devices on the spacecraft. Significant non-Maxwellian plasma distributions may also occur in nature as a mixture of ionospheric and magnetospheric plasmas or secondaries produced by photoionization in the thermosphere or auroral precipitation. The general formulas for current collection (volt-ampere curves) by planar, cylindrical, and spherical Langmuir probes in isotropic and anisotropic non-Maxwellian plasmas are examined. Examples are given of how one may identify and remove the non-Maxwellian components in the Langmuir probe current to permit the ionospheric parameters to be determined. Theoretical volt-ampere curves presented for typical examples of non-Maxwellian distributions include: two-temperature plasmas and a thermal plasma with an energetic electron beam. If the nonionospheric electrons are Maxwellian at a temperature distinct from that of the ionosphere electrons, the volt-ampere curves can be fitted directly to obtain the temperatures and densities of both electron components without resorting to techniques that attempt to derive the plasma distribution from the current by taking derivatives. For an arbitrary isotropic distribution, the current for retarded particles is shown to be identical for the three geometries. For anisotropic distributions, the three probe geometries are not equally suited for measuring the ionospheric electron temperature and density or for determining the distribution function in the presence of non-Maxwellian background electrons. (C) 1999 American Institute of Physics. [S0034-6748(99)02807-5]
\end{abstract}

\section{INTRODUCTION}

Langmuir probes have been used for many years on rockets and satellites to measure ionospheric electron temperature, $T_{e}$, and ion and electron number densities, $N_{i}$ and $N_{e}$, see references in Boyd and Twiddy ${ }^{1}$ and Brace. ${ }^{2}$ The Langmuir probe technique involves exposing a metallic collector to the plasma surrounding the vehicle, usually by mounting it on a boom that is longer than a Debye length at the lowest density expected to be encountered. The boom places the probe in contact with ionospheric plasma at a distance where the measurements are undisturbed by the presence of the vehicle. The probe current, $I$ (the sum of the ion and electron currents, $I_{i}$ and $I_{e}$ is measured as the collector voltage, $V$ ), is repeatedly swept through a suitable range with respect to the plasma potential. Analysis of the resulting $I-V$ curves usually yields the ionospheric parameters $T_{e}$ and $N_{e}$. Various sources of measurement error which affect this analysis were discussed by Brace, ${ }^{2}$ but that work did not cover the effects of nonionospheric or non-Maxwellian electron populations.

The measurements are very simple and direct when the electron energy distribution is essentially Maxwellian as is true nearly everywhere in the ionosphere. In this case, the electron energy can be characterized by a single scalar value, $T_{e}$. However, if additional electron populations are present in significant magnitude, the determination of $T_{e}$ is more complicated and may not be possible if the density of the additional component is sufficiently high. Sources of such additional populations may include: (1) photoelectrons produced in the daytime ionosphere in the process of forming the ionosphere, (2) degraded secondary electrons produced by precipitating auroral electrons and ions, (3) photoelectrons ejected from sunlit spacecraft surfaces, (4) secondaries produced by the impact at spacecraft velocities of thermospheric molecules, (5) secondaries emitted when the spacecraft potential is high enough to accelerate ionospheric ions or electrons to energies that exceed the ionization potential of spacecraft metallic surfaces, and (6) electron beams or plumes emitted from devices onboard the satellite. The geophysical sources (1 and 2), although present much of the time, usually have a negligible influence on the $I-V$ curves, except perhaps in regions of very low ionospheric density. The nongeophysical components (3-6) can have high enough densities to significantly distort the $I-V$ curves and affect the determination of $T_{e}$ by distorting the electron retarding regions of the $I-V$ curves. They may also affect the measurements of total ionospheric density, $N_{e}$ and $N_{i}$. In these cases, the ionospheric parameters (of the low energy electrons) can only be obtained by identifying the nonionospheric contributions to the $I-V$ curves and subtracting them out, or by fitting the $I-V$ curve with theoretical expressions which include the nonionospheric components. Examples of the effects of non-Maxwellian situations are: impact ionization on the Pioneer Venus Orbiter discussed by Whipple 
et al. $;^{3}$ an example of two-temperature distributions observed in the Venus ionotail described by Brace et al. $;^{4}$ and multicomponent superthermal electrons by Knudsen and Miller. ${ }^{5}$

This work presents the formulas for and examples of Langmuir probe current collection in such non-Maxwellian plasmas, and shows when the electron energy distribution can be derived from measurements of the first and second derivatives in the retarding region of the $I-V$ curves. Specific examples are also presented to illustrate the method of deriving ionospheric parameters from fitting the $I-V$ curves. The results show that different probe geometries are affected differently by the presence of non-Maxwellian components.

The equations are derived for probe-plasma conditions such that the sheath surrounding the probe may be considered large compared with the probe radius; this is the socalled orbital motion limited condition. This condition simplifies the equations and is valid for many past and future ionosphere measurements with Langmuir probes. Under conditions of sufficiently high plasma density at a given probe radius, the ratio of Debye length to probe radius will be too small to meet the orbital motion limited condition and the more general form of the probe equations should be used. The equations derived in the following sections can be modified to this more general case; however, this will not be pursued in this article.

\section{THE CONVENTIONAL LANGMUIR PROBE TECHNIQUE FOR MAXWELLIAN ELECTRONS}

A Langmuir probe may have any geometry, but cylindrical, spherical, and planar probes are usually employed because of their symmetry. The basis for $T_{e}$ measurements is the conventional Langmuir probe theory of Mott-Smith and Langmuir, ${ }^{6}$ assuming a Maxwellian energy distribution, $F(E)$, given by

$$
F(E) \propto \sqrt{E} \exp \left(\frac{-E}{k T_{e}}\right),
$$

where $k$ is the Boltzmann constant. Thus, in a Maxwellian plasma, when the probe to plasma potential, $V$, is driven negative, $I_{e}$ decreases exponentially

$$
I_{e}=I_{0} \exp \left(\frac{e V}{k T_{e}}\right),
$$

where $I_{0}$ is the random electron current given by $A N_{e} e\left(k T_{e} / 2 \pi m_{e}\right)^{1 / 2}$, where $e$ is the electron charge, $m_{e}$ is electron mass, and $A$ is the probe area. This equation for what is known as the electron retarding region in a Maxwellian plasma is the same for all probe geometries. It is shown below that this result has a generalization that the expression for the retarded current is identical for the three probe geometries for isotropic non-Maxwellian distributions. The derivation of $T_{e}$ is usually done by fitting the electron retarding region with an exponential function, and sometimes $T_{e}$ is obtained electronically by measuring the ratio of the first and second derivatives of $I_{e}$ rather than through analysis of the $I-V$ curves themselves. ${ }^{7,8}$
Next consider the ion and electron saturation regions of the $I-V$ curves. The form of the acceleration region current depends on the geometry of the collector and is approximated by

$$
I_{\text {accelerated }} \propto\left(1+\frac{e V}{k T}\right)^{p},
$$

where $p=0,1 / 2$, or 1 for planar, cylindrical, or spherical geometries, respectively. For satellite applications, Eq. (3) is modified to include the effects of the large spacecraft drift velocity relative to the essentially stationary ionospheric plasma. ${ }^{9}$ The general ion and electron acceleration current expressions are used to generate the $I-V$ curves that are shown in the examples rather than the Eq. (3) approximation.

Examples of the $I-V$ curves for the three geometries in purely Maxwellian plasmas are given in Figs. 1(a)-1(c) which show $I-V$ curves for four different temperatures. The calculation assumed $N_{e}=10^{5} \mathrm{~cm}^{-3}$, a collector area of 6.73 $\mathrm{cm}^{2}$ and a spacecraft velocity of $8 \mathrm{~km} / \mathrm{s}$. Note that the curves are downranged by a factor of 10 where necessary to allow the large electron acceleration currents to be shown on the same plot as the rest of curve. These figures illustrate the effect of $T_{e}$ on the width of the electron retardation regions.

For a Maxwellian plasma, all three probe geometries are suitable for the measurement of $T_{e}$. However, the electron saturation regions differ greatly with collector geometry. Note that the electron saturation region of the cylindrical probe is nearly independent of $T_{e}$, whereas this region is highly temperature dependent for the planar and spherical probes. This illustrates a practical advantage of the cylindrical probe; i.e., that a fixed probe potential can be applied to make continuous measurements of $N_{e}$ without pausing to measure $T_{e}$, assuming a known and stable value for the potential of the spacecraft. The cylindrical and spherical probes have the advantage of producing large saturation currents for the same density, an advantage for measurements in regions of very low density.

\section{MULTICOMPONENT PLASMAS AND OTHER NON- MAXWELLIAN DISTRIBUTIONS}

When nonionospheric (non-Maxwellian) electrons are present in detectable quantities, the standard Langmuir equations may lead to significant errors in the measurements of $N_{e}$ and $T_{e}$. To handle these situations, we examine the current equations for arbitrary electron energy distributions for the three probe geometries. These equations are integrals over the velocity distribution functions (VDF) for multicomponent populations of ions and electrons. The discussion begins with formulas for general anisotropic VDFs, then considers the general isotropic case, and finally, the anisotropic case for a Maxwell-Boltzmann distribution. The effects of these nonionospheric populations are illustrated by calculating $I-V$ curves for a two temperature distribution as an example of the isotropic case, and an energetic electron beam with a superposed Maxwell-Boltzmann distribution as an example of the anisotropic case. The information that can be obtained about the electron distribution functions by using 

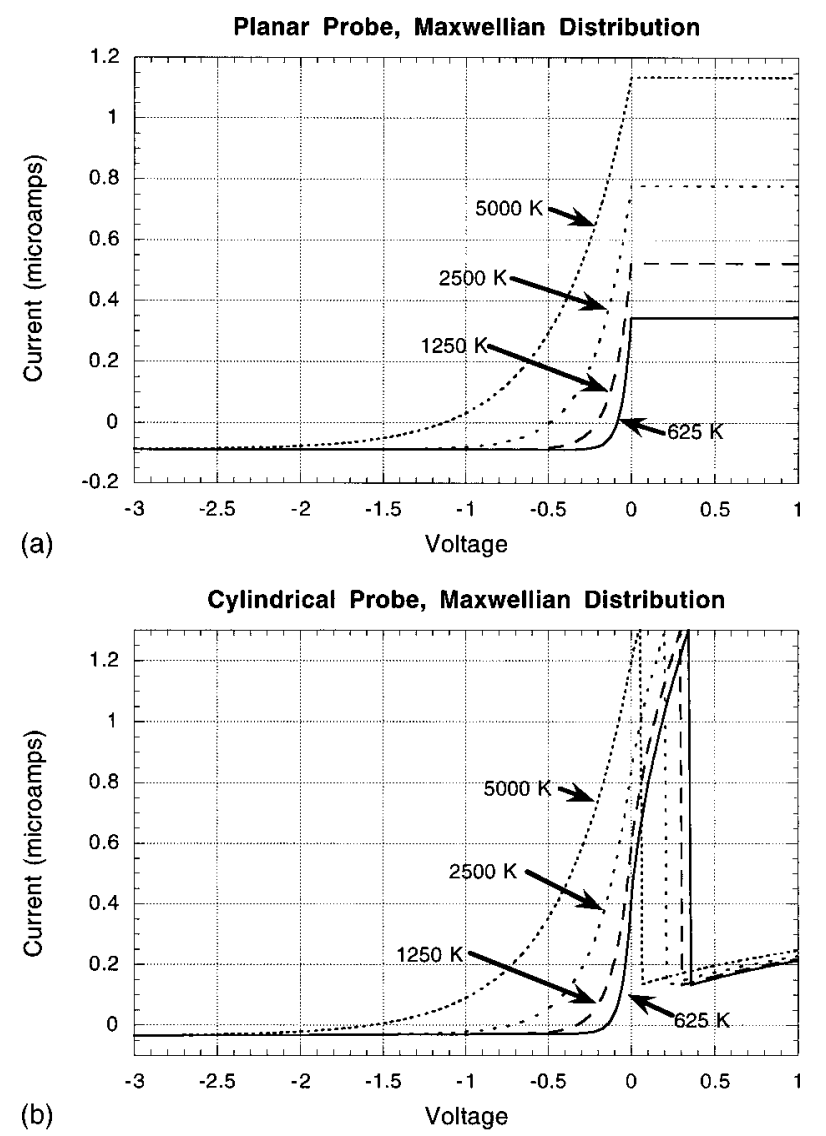

(b)

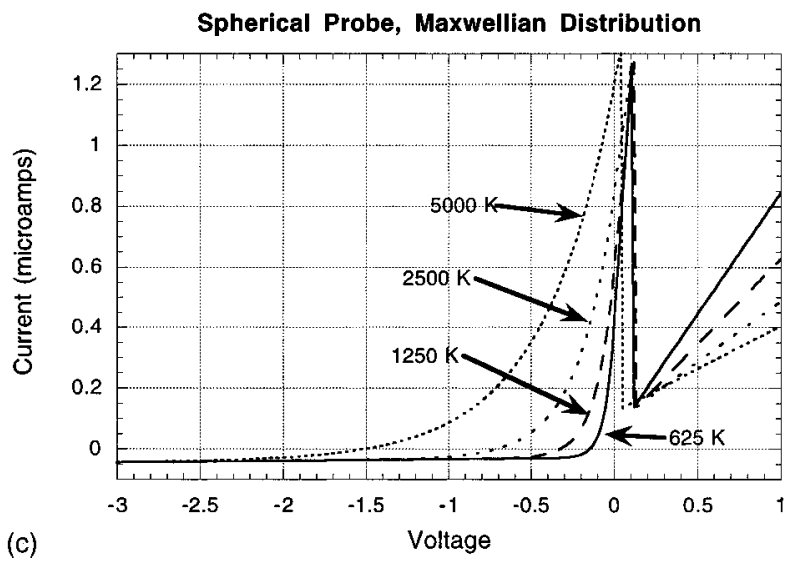

FIG. 1. (a) Planar probe curves computed for a Maxwellian plasma illustrate the effect of $T_{e}$ on the width of the retarding region. Note that the electron saturation region is flat because the calculation assumes an infinite plane; i.e., a perfectly guarded planar probe. The amplitude of the electron saturation current varies greatly with $T_{e}$. (b) Same as (a) but for a cylindrical probe. Note that the electron saturation currents are nearly independent of $T_{e}$. (c) Same as (a) but for a spherical probe.

the first and second derivatives of the $I-V$ curves is discussed, and later fits are made to the curves to illustrate obtaining the ionosphere parameters.

\section{CURRENT EQUATIONS FOR ARBITRARY VELOCITY DISTRIBUTIONS}

The general form of the VDF for the $s$ th specie ( $s$ is an electron or an ion specie) divided into distinct populations $j$ is a sum over the $j$ populations:

$$
F_{s}(u, v, w)=\sum_{j} n_{s}^{j} F_{s}^{j}(u, v, w),
$$

where $n_{s}^{j}$ is the number density of population $j$ for specie $s$, $F_{s}^{j}$ is the individual VDF, and $u, v, w$ are velocities in the $x, y, z$ directions. The sum of the number densities over the $j$ populations is the total number density of specie $s, \sum_{j} n_{s}^{j}=n_{s}$, and the VDFs are normalized to unity,

$$
\int_{-\infty}^{\infty} \int_{-\infty}^{\infty} \int_{-\infty}^{\infty} d u d v d w F_{s}^{j}(u, v, w)=1
$$

Two classes of distributions: isotropic and anisotropic are considered. For the isotropic VDF, the general form is

$$
F_{s}^{j}(u, v, w)_{\text {isotropic }}=G_{s}^{j}\left(u^{2}+v^{2}+w^{2}\right),
$$

where $G$ is a function of the sum of velocities squared or of the energy. The most applicable form for a collision dominated plasma is the Maxwell distribution function:

$$
\begin{aligned}
& G_{\text {Maxwell }}\left(u^{2}+v^{2}+w^{2}\right) \\
& \quad=\left(\frac{m}{2 \pi k T}\right)^{3 / 2} \exp \left(-\frac{m\left(u^{2}+v^{2}+w^{2}\right)}{k T}\right),
\end{aligned}
$$

where $m$ and $T$ are the mass and temperature of the $j$ th component of the $s$ th specie (for simplicity, the subscripts and superscripts are not explicitly written in all cases).

The anisotropic distribution has a preferred direction in space and could result, for example, from a beam of electrons induced by an acceleration process, or from rapid motion of the probe through the plasma, or from an anisotropy in the temperature of the electrons or ions (electrons parallel and perpendicular to the magnetic field can have distinct temperatures). Anisotropic $T_{e}$ was first measured by Clark et al. ${ }^{10}$ and theoretically predicted by Schunk and Watkins. ${ }^{11}$ We will employ a specific form of the anisotropic VDF which applies to many space plasma situations:

$$
\begin{aligned}
& F_{s}^{j}(u, v, w)_{\text {anisotropic }} \\
& \quad=F_{s}^{j}\left[a\left(u-u_{0}\right), b\left(v-v_{0}\right), c\left(w-w_{0}\right)\right]_{\text {isotropic }},
\end{aligned}
$$

where $a, b$, and $c$ are constants that could represent temperature anisotropy, and $u_{0}, v_{0}, w_{0}$ represent a drift velocity of the plasma relative to the probe. An applicable form of the anisotropic VDF is the Maxwell-Boltzmann distribution which is given by Eq. (7) with the sum of squares of the velocities replaced by the sum of squares with respect to the drift velocity,

$$
a^{2}\left(u-u_{0}\right)^{2}+b^{2}\left(v-v_{0}\right)^{2}+c^{2}\left(w-w_{0}\right)^{2} .
$$

Another useful form for the anisotropic VDF is as a spherical harmonic expansion.

\section{A. General current equations}

The Langmuir probe current equations are given below for the three standard geometries; planar, cylindrical, and spherical. To simplify the formulas we assume large symmetrical sheaths and orbital-motion-limited collection for the cylindrical and spherical probes, which is valid over most ionosphere conditions as long as the relevant probe dimen- 
sion is smaller than a few $\mathrm{cm}$. It will become apparent that each geometry has advantages and disadvantages which depend upon the type of non-Maxwellian distribution.

\section{Planar probe general formulas}

For a planar probe of area $A$, the general formula for the current is obtained from the current perpendicular to the probe, $A n_{s}^{j} q_{s}^{j} u d u F(u, v, w) d v d w$, integrated over all velocities parallel to the plane ( $v$ and $w$ ) and over velocities directed into the plane $(u)$ that are greater than the retarding potential,

$$
i_{\text {plane }}=A \sum_{s, j} n_{s}^{j} q_{s}^{j} \int_{0, \sqrt{-2 q V / m}}^{\infty} u d u F_{s}^{j}(u)_{1 \mathrm{D}}
$$

where $q$ is the charge, $m$ the mass of the $s$ th specie and $j$ th population. The lower limits on the integral correspond to accelerated $(q V>0)$, retarded $(q V<0)$ particles, respectively, $u$ is the component of velocity perpendicular to the planar probe. And $F_{1 \mathrm{D}}$ is the one-dimensional or " $1 \mathrm{D}$ " distribution, defined by the integral over velocities parallel to the probe surface,

$$
F(u)_{1 \mathrm{D}} \equiv \int_{-\infty}^{\infty} \int_{-\infty}^{\infty} d v d w F(u, v, w),
$$

and where the subscripts and superscripts are dropped for simplicity of notation. This result is general for any VDF, isotropic or anisotropic. In the ionosphere, the electron current at zero probe potential is about a factor of 170 greater than the ion current because of the large ion/electron mass ratio, hence the $I-V$ characteristic is dominated much more by the electron current than the ion current. Therefore, the total current in the retarding region can be used to attempt to determine the electron velocity distribution. We adopt the convention that the electron current is positive. Thus the first derivative of the planar electron current is given by

$$
\frac{d i_{\text {plane }}}{d e V}=\frac{A e}{m_{e}} \sum_{j} n^{j} F^{j}\left(\sqrt{\frac{-2 e V}{m_{e}}}\right)_{1 \mathrm{D}},
$$

where $e V<0$. The derivative is proportional to the 1D VDF. This result does not imply that the individual populations $j$ can be obtained from the first derivative which gives the sum of the populations. However, if the populations dominate in distinct energy regions, then the derivative can be used to determine the dominant populations. Also the 1D VDF is not the same as the original 3D VDF of Eq. (4), however, it is a useful concept. Later in the discussion on isotropic plasmas, the relationship between the 1D and 3D distributions is examined. In general, the second derivative of the planar electron current appears to be not related directly to the distribution function, since it is the first derivative of the 1D VDF. However, if the distribution takes the specific form of anisotropic VDF that we assumed in Eq. (8), then it can be shown using integration by parts, that

$$
\begin{aligned}
\frac{d^{2} i_{\text {plane }}}{d e V^{2}}= & \frac{2 \pi A e}{m_{e}^{2}} \sum_{j} \frac{\left(\sqrt{\frac{-2 e V}{m_{e}}}-u_{0}^{j}\right)}{\sqrt{\frac{-2 e V}{m_{e}}}} \\
& \times \frac{a^{2}}{b c} n^{j} G^{j}\left[a^{2}\left(\sqrt{\frac{-2 e V}{m_{e}}}-u_{0}^{j}\right)^{2}\right]_{3 \mathrm{D}},
\end{aligned}
$$

where $a, b, c$ are anisotropy parameters which are distinct for each population, and $u_{0}$ is the population dependent drift velocity in the direction perpendicular to the planar probe surface. Thus, the second derivative of the planar current is equal to a weighted sum of the 3D VDFs for our assumed form of the anisotropic distribution. If the populations have distinct energy domains, then it may be possible to obtain some information on the VDF of each of the populations. The result is independent of the drift velocities in the directions along the probe surface, consistent with the planar probe sensing the $1 \mathrm{D}$ distribution. The planar probe current was treated by Federov ${ }^{12}$ for the anisotropic distribution expanded in spherical harmonics. In the special case of an isotropic $\operatorname{VDF}\left(a=b=c=1, u_{0}=0\right)$, then Eq. (12) reduces to the sum over the individual 3D VDFs.

\section{Cylindrical probe general formulas}

For a cylindrical probe with an anisotropic VDF, the general formula for the current is more complicated than for a planar probe. The geometry is no longer rectilinear and we must integrate over the circumference of the cylindrical surface which changes the direction of the normal and tangential velocities relative to the anisotropy direction in space. If we let the $z$ axis be along the probe axis, the dependence on a drift velocity, $w_{0}$ in the $z$ direction is eliminated. We use our general form for the anisotropic VDF, given by Eqs. (6) and (8) and define a 2D VDF,

$$
F\left[a\left(u-u_{0}\right), b\left(v-v_{0}\right)\right]_{2 \mathrm{D}} \equiv \int_{-\infty}^{\infty} d w F(u, v, w)_{\text {anisotropic }},
$$

where $F_{\text {anisotropic }}$ is given by Eq. (8). At an arbitrary position $\theta$ along the probe circumference, the radial, $u^{\prime}$, and tangential, $v^{\prime}$, velocities are related to the $x, y$ components $u, v$ by the transformation,

$$
u=u^{\prime} \cos \theta-v^{\prime} \sin \theta, \quad v=u^{\prime} \sin \theta+v^{\prime} \cos \theta .
$$

The orbital-motion-limited cylinder probe current is adapted from the derivations of Mott-Smith and Langmuir, ${ }^{6}$

$$
\begin{aligned}
i_{\text {cylinder }}= & 2 A \sum_{s, j} n_{s}^{j} q_{s}^{j} \frac{1}{2 \pi} \int_{0}^{2 \pi} d \theta \int_{0, \sqrt{-2 q V / m}}^{\infty} u d u \\
& \times \sqrt{u^{2}+\frac{2 q V}{m}} F_{s}^{j}\left[a\left(u \cos \theta-u_{0}\right),\right. \\
& \left.b\left(u \sin \theta-v_{0}\right)\right]_{2 \mathrm{D}},
\end{aligned}
$$

where $A$ is the cylinder area and transformation (14) was used in Eq. (13), $u^{\prime}$ is relabeled $u$ and we may set $v^{\prime}=0$. The integral is complicated and involves integration over a 
modified Bessel function in the case of a MaxwellBoltzmann VDF. The first and second derivatives of the cylindrical probe current in general do not directly give the distribution function. However, Federev ${ }^{12}$ has shown that the cylindrical $I-V$ curve can yield information on the anisotropic distribution function.

\section{Spherical probe general formulas}

For the spherical Langmuir probe, there is no preferred direction and the current is an integral over $4 \pi$ directions on the sphere. The general rotation transformation of velocities from the fixed $x, y, z$ velocities, $u^{\prime}, v^{\prime}, w^{\prime}$ to velocities on the sphere at the position given by the spherical polar angles, $\theta, \phi$ with $u$ the radial velocity and $v$ and $w$ the tangential velocities, is given by

$$
\begin{aligned}
& u=u^{\prime} \cos \theta+\sin \theta\left(v^{\prime} \cos \phi+w^{\prime} \sin \phi\right), \\
& v=-u^{\prime} \sin \theta+\cos \theta\left(v^{\prime} \cos \phi+w^{\prime} \sin \phi\right), \\
& w=-v^{\prime} \sin \phi+w^{\prime} \cos \phi,
\end{aligned}
$$

where $\theta, \phi$ are the polar and azimuthal angles, respectively. The inverse transformation is the transpose of Eq. (16). Then the spherical probe current in the limit of a large sheath (orbital-motion-limited case) is given by ${ }^{5}$

$$
\begin{aligned}
i_{\text {sphere }}= & \frac{A}{4} \sum_{s, j} n_{s}^{j} q_{s}^{j} \int_{0}^{\pi} \sin \theta d \theta \int_{0}^{2 \pi} d \phi \\
& \times \int_{0, \sqrt{-2 q V / m}}^{\infty} u d u\left(u^{2}+\frac{2 q V}{m}\right) \cdot F_{s}^{j}\left[a\left(u \cos \theta-u_{0}\right),\right. \\
& \left.b\left(u \sin \theta \cos \phi-v_{0}\right), c\left(u \sin \theta \sin \phi-w_{0}\right)\right]
\end{aligned}
$$

where $A$ is the sphere area, and $F^{j}$ is our general anisotropic 3D VDF given by Eq. (8). For retarded electrons, the first derivative is

$$
\begin{aligned}
\frac{d i_{\text {sphere }}}{d e V}= & \frac{A}{4} \sum_{j} n_{e}^{j} e \frac{2}{m_{e}} \int_{0}^{\pi} \sin \theta d \theta \int_{0}^{2 \pi} d \phi \\
& \times \int_{0, \sqrt{-2 e V / m_{e}}}^{\infty} u d u F^{j},
\end{aligned}
$$

and the second derivative is given by

$$
\begin{aligned}
\frac{d^{2} i_{\text {sphere }}}{d e V^{2}}= & \frac{A}{4} \sum_{j} n_{e}^{j} e \frac{2}{m_{e}^{2}} \int_{0}^{\pi} \sin \theta d \theta \\
& \times\left.\int_{0}^{2 \pi} d \phi F^{j}\right|_{u=\sqrt{-2 e V / m_{e}}} .
\end{aligned}
$$

Thus, the second derivative of the spherical probe current is the average over all orientations of the anisotropic distribution function, or it yields the isotropic part of the distribution as was shown by Federov. ${ }^{12}$

\section{B. Current equations for isotropic distribution of electrons}

The isotropic velocity distribution function has the general form given by Eq. (6). We now demonstrate that, for isotropic electron populations, the three geometries have
$I-V$ curves with identical functional form for the retarded current. Thus, for all three geometries, the first derivative yields the 1D VDF and the second derivative yields the 3D VDF. This result means that theoretically all three are suitable for measuring isotropic, non-Maxwellian electron velocity distributions.

\section{Standard form of the isotropic, retarded current}

Given the form of the isotropic VDF, Eq. (6), the 1D distribution can be rewritten as

$$
G_{1 \mathrm{D}}\left(u^{2}\right)=2 \pi \int_{0}^{\infty} t d t G\left(u^{2}+t^{2}\right),
$$

where $G$ is the $3 \mathrm{D} \mathrm{VDF}$ and the polar transformation of variables was used, $v=t \cos \alpha, w=t \sin \alpha$. Thus the planar current can be rewritten as

$$
i_{p}^{\text {isotropic }}=A \sum n q \int_{0, \sqrt{-X}}^{\infty} u d u 2 \pi \int_{0}^{\infty} t d t G\left(u^{2}+t^{2}\right),
$$

where $X=2 q V / m$; the lower limit 0 is for accelerated particles when $X>0$, and the limit $\sqrt{-X}$ is for retarded particles when $X<0$. The accelerated current is independent of voltage, and thus we consider only the retarded particles which yield information on the VDF. With a change of variables, the retarded planar current expression is

$$
i_{p}^{\text {isotropic }}{ }_{\text {retarded }}=A \sum n q \pi \int_{0}^{\infty} s^{3} d s G\left(s^{2}-X\right) .
$$

The cylindrical current is obtained by substituting an isotropic VDF in Eq. (15), giving the form,

$$
\begin{aligned}
i_{c}^{\text {isotropic }}= & A \sum 2 n q \int_{0, \sqrt{-X}}^{\infty} u d u \sqrt{u^{2}+X} \\
& \times \int_{-\infty}^{\infty} d w G\left(u^{2}+w^{2}\right) .
\end{aligned}
$$

Successive transformation of variables, $u^{2}+X=t^{2}$, and $t$ $=\sin \theta s, w=\cos \theta s$, and the integral over $\theta$ yields $\pi / 2$, and thus the retarded cylindrical current is given by the integral in Eq. (22), $i_{c}^{\text {isotropic }}{ }_{\text {retarded }}=i_{p}^{\text {isotropic }}$ retarded .

The spherical current, Eq. (17), for an isotropic distribution has the form,

$$
i_{s}^{\text {isotropic }}=A \sum n q \pi \int_{0, \sqrt{-X}}^{\infty} u d u\left(u^{2}+X\right) G\left(u^{2}\right),
$$

and using the transformation, $u^{2}+X=s^{2}$, the spherical probe retarded current is found to have the form of Eq. (22), or, $i_{s}^{\text {isotropic }}{ }_{\text {retarded }}=i_{p}^{\text {isotropic }}$ retarded. Thus the three probe geometries have the identical retarded current expression for an isotropic VDF.

\section{First derivative of the isotropic retarded current}

The first derivative of the retarded current was shown in Eq. (11) to be given by the 1D distribution for a planar probe, thus for isotropic plasma, the three probe geometries all have the identical form given by 


$$
\frac{d i^{\text {isotropic }}}{d e V}=A \sum \frac{n_{e} e}{m_{e}} G_{1 \mathrm{D}}\left(-\frac{2 e V}{m_{e}}\right),
$$

where the 1D VDF is given by Eq. (20).

Examine the relation of the 1D distribution to the full 3D VDF. The 3D VDF can be expanded in Laguerre polynomials, ${ }^{13}$

$$
G(x)=e^{-x} \sum_{n=0}^{\infty} g_{n} L_{n}^{(0)}(x),
$$

then the 1D VDF is the integral,

$$
G_{1 \mathrm{D}}(x)=\pi \int_{0}^{\infty} d s G(s+x),
$$

which can be rewritten, using the relations among Laguerre polynomials given by Rainville, ${ }^{13}$ as follows:

$$
G_{1 \mathrm{D}}(x)=e^{-x} \pi \sum_{n=0}^{\infty} g_{n} L_{n}^{(-1)}(x) .
$$

For a Maxwell distribution, $g_{n}=0$ for $n>0$, which is the only case when the 1D and 3D distributions have the same functional form. The first few terms of the two distributions are given by

$$
\begin{aligned}
& G(x)=e^{-x}\left[g_{0}+g_{1}(1-x)+g_{2}\left(1-2 x+\frac{x^{2}}{2}\right)+\cdots\right], \\
& G_{1 \mathrm{D}}(x)=\pi e^{-x}\left[g_{0}-g_{1} x+g_{2}\left(-x+\frac{x^{2}}{2}\right)+\cdots\right] .
\end{aligned}
$$

The distributions are distinct, but the dominant terms in $x$ are identical.

\section{Second derivative of isotropic retarded current}

The second derivative of the isotropic current is readily obtained from Eq. (12) by substituting the isotropic distribution, we obtain

$$
\frac{d^{2} i^{\text {isotropic }}}{d e V^{2}}=\frac{2 \pi}{m_{e}^{2}} A \sum n_{e} e G\left(\frac{-2 e V}{m_{e}}\right) .
$$

A result first found earlier by Mott-Smith and Langmuir. ${ }^{6}$ In the next section, we give the current formulas for the special anisotropic case of the Maxwell-Boltzmann distribution with isotropic temperatures.

\section{The Maxwell-Boltzmann anisotropic case}

The VDF for a Maxwell-Boltzmann (MB) distribution is given by the formula,

$$
\begin{aligned}
F_{s}^{j}(u, v, w)_{\mathrm{MB}}= & \left(\frac{m_{s}^{j}}{2 \pi k T_{s}^{j}}\right)^{3 / 2} \exp \left\{-\frac{m_{s}^{j}}{2 k T_{s}^{j}}\left[\left(u-u_{0}\right)^{2}\right.\right. \\
& \left.\left.+\left(v-v_{0}\right)^{2}+\left(w-w_{0}\right)^{2}\right]\right\},
\end{aligned}
$$

where the drift velocity components $u_{0}, v_{0}, w_{0}$ may be different for each specie and population of a specie. For simplicity, we will assume below that all species have the same drift velocity.

\section{Planar probe equations for anisotropic MB distribution}

The 1D VDF is obtained by substituting Eq. (34) in Eq. (12),

$$
\left.F_{s}^{j}(u)_{\mathrm{MB}}\right|_{1 \mathrm{D}}=\left(\frac{m_{s}^{j}}{2 \pi k T_{s}^{j}}\right)^{1 / 2} \exp \left[-\frac{m_{s}^{j}}{2 k T_{s}^{j}}\left(u-u_{0}\right)^{2}\right],
$$

which leads to the current expressions,

$$
i_{\text {planar }_{\mathrm{MB}_{\text {accelerated }}}}=\sum_{j, s} i_{\text {random }}\left[\exp \left(-r^{2}\right)+\sqrt{\pi} r \operatorname{erfc}(-r)\right]
$$

for accelerated particles, and

$$
\begin{aligned}
i_{\text {planar }_{\mathrm{MB}_{\text {retarded }}}}= & \sum_{j, s} i_{\text {random }}\left\{\exp \left[-(\sqrt{-\eta}-r)^{2}\right]\right. \\
& +\sqrt{\pi} r \operatorname{erfc}(\sqrt{-\eta}-r)\},
\end{aligned}
$$

for retarded particles, where $r$ is the ratio of drift velocity to thermal velocity,

$$
r_{s}^{j}=\frac{u_{0_{s}^{j}}}{\sqrt{2 k T_{s}^{j} / m_{s}^{j}}},
$$

and $\eta$ is the ratio of voltage to thermal energy,

$$
\eta_{s}^{j}=\frac{q_{s}^{j} V}{k T_{s}^{j}},
$$

and the random current, the current due only to thermal motion of the particles, is

$$
i_{\text {random }_{s}^{j}}=A n_{s}^{j} \sqrt{\frac{k T_{s}^{j}}{2 \pi m_{s}^{j}}} .
$$

\section{Cylindrical probe equations for anisotropic MB distribution}

Using the Maxwell-Boltzmann distribution (32) in the expression for the cylindrical probe current, Eq. (15), and transforming the drift velocities, we find

$$
\begin{aligned}
i_{\text {cylinder }}= & \sum_{s, j} i_{\text {random }} \frac{4}{\sqrt{\pi}} \int_{0, \sqrt{-\eta}}^{\infty} t d t \sqrt{t^{2}+\eta} \\
& \times \exp \left[-\left(t^{2}+r^{2}\right)\right] I_{0}(2 t r),
\end{aligned}
$$

which formula cannot be evaluated in simple analytical form. $I_{0}$ is the modified Bessel function and $r$ and $\eta$ are given above. Useful asymptotic approximations have been obtained for this integral by Hoegy and Wharton. ${ }^{9}$ However, since we are evaluating the cylinder current for a wide range of values of $r$ and $\eta$, we evaluate it numerically using the fast, efficient routines from Numerical Recipes. ${ }^{14}$ The limit of Eq. (38) for zero drift velocity or the isotropic Maxwell distribution gives for accelerated particles,

$$
i_{\text {random }}\left[\frac{2}{\sqrt{\pi}} \sqrt{\eta}+\exp (\eta) \operatorname{erfc}(\sqrt{\eta})\right],
$$


for large $\eta$, this expression is approximated by $(2 / \sqrt{\pi}) \sqrt{1+\eta}$. For retarded particles, the current is given by

$$
i_{\text {random }} \exp (\eta)
$$

\section{Spherical probe equations for anisotropic MB distribution}

The Maxwell-Boltzmann distribution (32) in the expression for the spherical probe current, Eq. (17), gives the expression,

$$
\begin{aligned}
i_{\text {sphere }}= & \sum_{s, j} i_{\text {random }} \frac{1}{2 r} \int_{0, \sqrt{-\eta}}^{\infty} d t\left(t^{2}+\eta\right) \\
& \times\left\{\exp \left[-(t-r)^{2}\right]-\exp \left[-(t+r)^{2}\right]\right\} .
\end{aligned}
$$

The spherical geometry, results in spherical Bessel functions which can be integrated to give,

$$
\begin{aligned}
i_{\text {sphere }_{\text {accelerated }}}= & \sum_{s, j} i_{\text {random }}\left[\frac{1}{2} \exp \left(-r^{2}\right)\right. \\
& \left.+\frac{\left(\eta+r^{2}+\frac{1}{2}\right)}{r} \frac{\sqrt{\pi}}{2} \operatorname{erf}(r)\right],
\end{aligned}
$$

for accelerated particles, and

$$
\begin{aligned}
i_{\text {sphere }_{\text {retarded }}}= & \sum_{s, j} i_{\text {random }} \frac{1}{2 r}\left\langle\left(\eta+r^{2}+\frac{1}{2}\right)(\sqrt{\pi} / 2)\right. \\
& \times[\operatorname{erf}(\sqrt{-\eta}+r)-\operatorname{erf}(\sqrt{-\eta}-r)] \\
& +\frac{(\sqrt{-\eta}+r)}{2} \exp \left[-(\sqrt{-\eta}-r)^{2}\right] \\
& \left.-\frac{(\sqrt{-\eta}-r)}{2} \exp \left[-(\sqrt{-\eta}+r)^{2}\right]\right\rangle,
\end{aligned}
$$

for retarded particles. In the limit of zero drift velocity, or an isotropic Maxwell distribution, Eq. (40a) reduces to

$$
i_{\text {random }}(\eta+1),
$$

and Eq. (40b) reduces to

$$
i_{\text {random }} \exp (\eta) \text {. }
$$

To aid the reader in visualizing the effects of nonMaxwellian electron distributions on the $I-V$ curves, Sections $\mathrm{V}$ and VI show theoretical $I-V$ curves for mixtures of electron populations having different temperatures and density ratios. $I-V$ curves are also shown for mixtures of thermal electrons and energetic beam electrons.

\section{I- $V$ CHARACTERISTICS FOR TWO-TEMPERATURE DISTRIBUTIONS}

Figures 2(a), 2(b), and 2(c) show planar, cylindrical and spherical probe $I-V$ curves for various density ratios of 10000 and $1000 \mathrm{~K}$ electron populations, with a combined total density of $10^{5} \mathrm{~cm}^{-3}$. Curves are shown for energetic component densities relative to the total density of 0,20 , 33.3 , and $50 \%$. $0 \%$ corresponds to the complete absence of the higher temperature component.
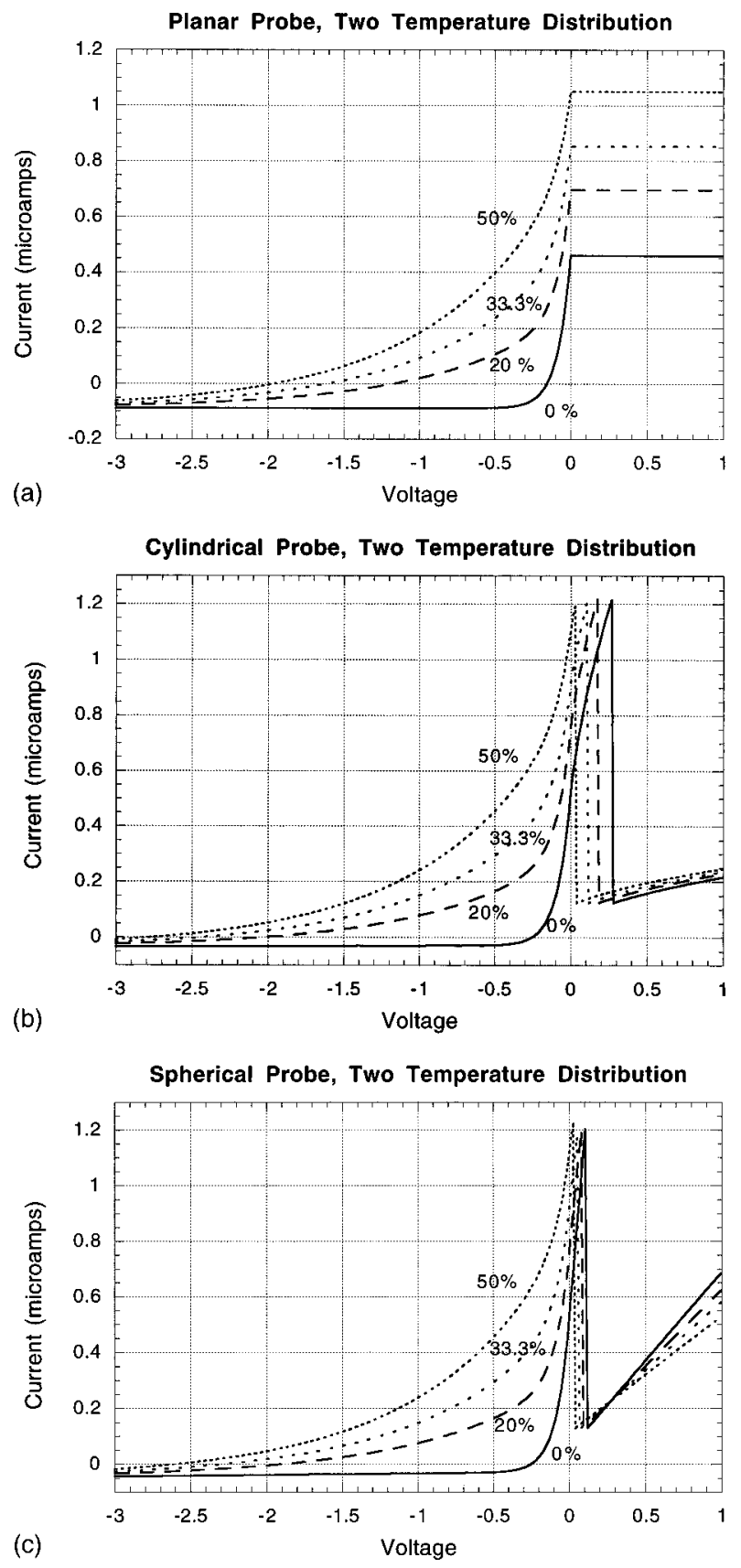

FIG. 2. (a) Planar probe curves for mixtures of electron populations having temperatures of 1000 and $10000 \mathrm{~K}$. The curves are labeled with the percentage of the total density represented by the higher temperature component. The low temperature component dominates the shape of the curves at low retarding potentials, while the high temperature component dominates the more negative end of the retarding region. (b) Same as (a), except for a cylindrical probe. (c) Same as (a), except for a spherical probe.

The higher temperature component enhances the current at greater retarding voltages as seen by comparing these figures with Figs. 1(a)-1(c). The temperatures and densities of the two components can be obtained by fitting a twotemperature exponential to the curves or by fitting the near and far retarding regions with single-temperature exponential. The latter procedure was used successfully in the fitting of $I-V$ curves from the PVO Langmuir probe which exhibited two-temperature characteristics in certain regions of the Venus ionotail. ${ }^{4}$ 


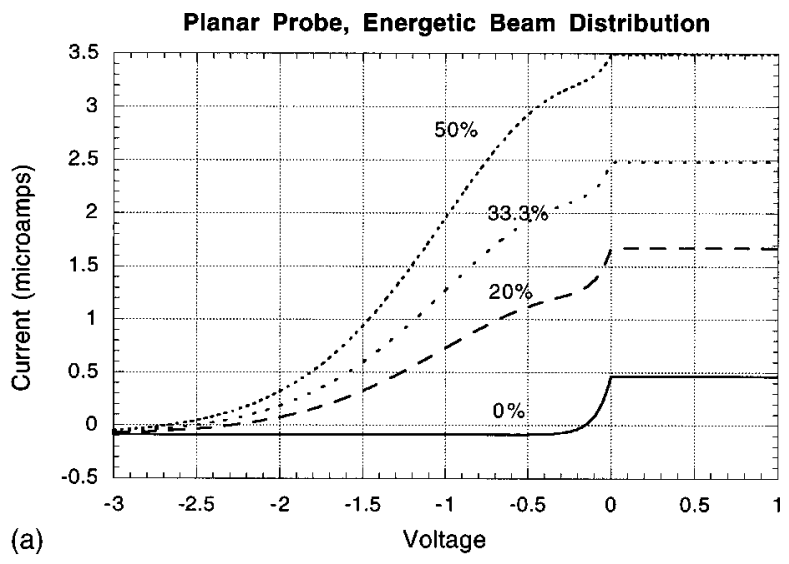

(a)

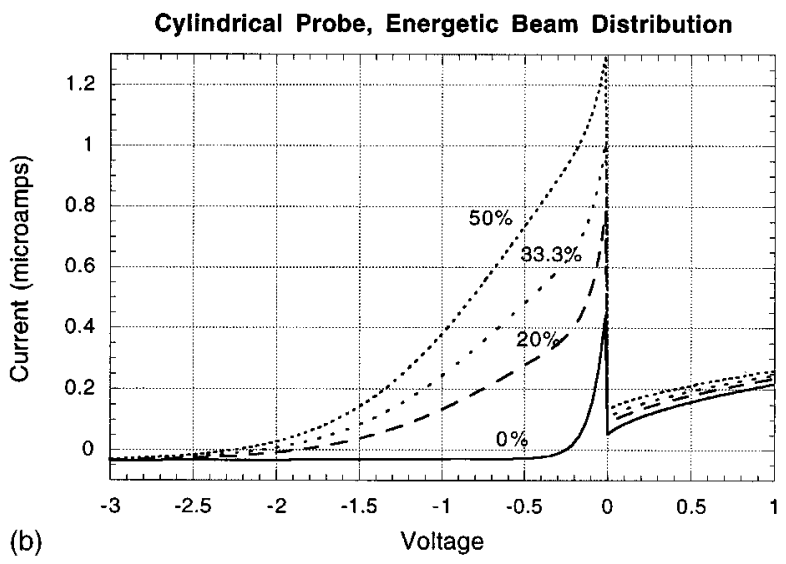

(b)

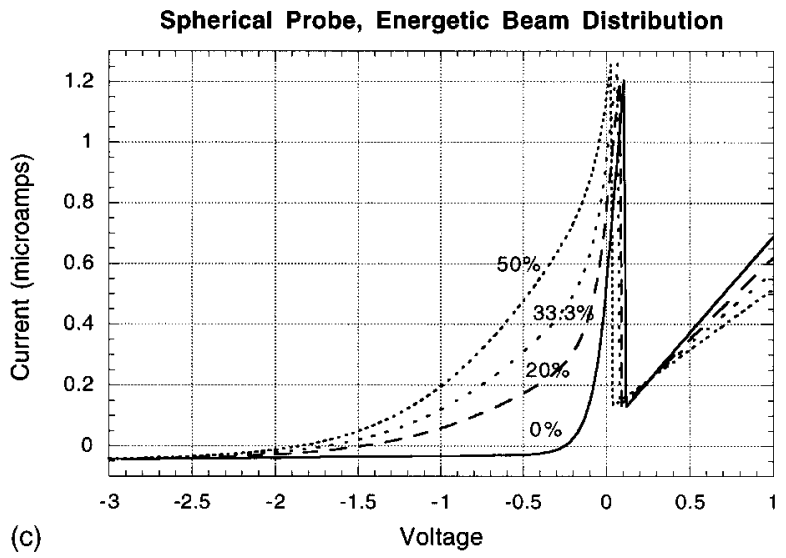

FIG. 3. (a) Planar probe curves for a $1 \mathrm{eV}$ energetic beam with a $2000 \mathrm{~K}$ energy spread and a $1000 \mathrm{~K}$ thermal component. The curves are labeled with the percentage of the total density represented by the beam. Note that the beam component dominates the retarding region for relatively small percentages of beam density, thus potentially interfering with the measurement of the cold ionospheric component. (b) Same as (a), except for a cylindrical probe. The retarding region is not as sensitive to the beam component as was the case for the planar probe (a). Also note that the electron saturation currents to a cylinder are relatively less sensitive to the presence of a beam than are planar probes and spherical probes (c). (c) Same as (a), except for a spherical probe. The retarding region is less sensitive to the beam component than either the planar or cylindrical probe [(a) and (b)] because thermal electrons are collected from $4 \pi$ radians, while the beam approaches the collector from only one direction.

\section{I- $V$ CHARACTERISTICS FOR THERMAL ELECTRONS PLUS AN ENERGETIC BEAM}

Figures 3(a)-3(c) show theoretical $I-V$ curves for combinations of $1000 \mathrm{~K}$ thermal electrons and a $1 \mathrm{eV}$ energetic beam having a $2000 \mathrm{~K}$ thermal spread. The assumed total density is $10^{5} \mathrm{~cm}^{-3}$. The beam is directed normal to the planar probe surface and perpendicular to the cylindrical probe axis. The beam direction is irrelevant for the spherical probe. This case is our example of an anisotropic distribution. The nonexponential nature of the curves shows immediately that the energy distribution is non-Maxwellian.

These examples are illustrative of the effects of nonMaxwellian plasmas on Langmuir probe measurements. Other combinations of secondaries, energetic beams and ionospheric electrons would change the shapes and amplitudes of the Langmuir probe characteristics in different and unique ways. The question remains as to how the ambient ionospheric temperature and density can be derived when such additional electron populations are present as an alternative to the derivative technique. The next section illustrates what can be learned about such complex distributions by fitting the $I-V$ curves using either one or two temperature distributions.

\section{FITTING $I-V$ CURVES FOR ONE- OR TWO- TEMPERATURE MAXWELLIAN DISTRIBUTIONS}

If one knew the form of the energy distributions of the secondary electron populations, one could in principle fit the $I-V$ curves for the energy and density of each of the contributions. In practice this is difficult, since one usually only has the $I-V$ curves themselves to work with. After finding that a single-temperature fit fails to achieve a sufficiently small standard deviation, one could proceed by trial and error to add various other components to the fit to improve the quality of the fit. The first step is usually to add another Maxwellian component at a suitable temperature and density. If such a two-temperature distribution fails to achieve an acceptable fit, one can proceed to add or substitute directed energetic beams with appropriate energies and temperatures of the type illustrated above in Sec. VI.

In this section, we go part way down this path by showing how well single- and two-temperature probe theories fit the $I-V$ curves shown in Figs. 1-3. We fitted the theoretical curves shown in Figs. 1-3 using techniques similar to those used in the analysis of Langmuir probe measurements from Pioneer Venus Orbiter ${ }^{4}$ and Dynamics Explorer-2. ${ }^{15}$ The retarding regions were fit first to a single Maxwellian. In some cases, the curves were then fit using a two-temperature Maxwellian to explore the feasibility of obtaining information on the nature of the distributions and to recover the ambient parameters.

The single-temperature fits were made using the simple form:

$$
I=a+b V+c \exp \left(\frac{11604.45 V}{T_{1}}\right),
$$

where $V$ is the probe voltage in volts, $T_{1}$ is the single temperature in $\mathrm{K}$, and $a, b$, and $c$ are constants to be determined by the fit. The first two terms represent the ion current amplitude and slope, respectively. The third term represents the retarded electron current. The fitting procedure steps through a range of temperatures $T_{1}$ while performing a least-squares fit of Eq. (42) to the theoretical curves to determine the co- 
TABLE I. $T_{e}$ and STD for single- and two-temperature fits to curves produced by a two-temperature distribution at 1000 and $10000 \mathrm{~K}$.

\begin{tabular}{lcrc}
\hline \hline \multicolumn{1}{c}{ ratio $n_{2} / n_{1}$} & 0.25 & 0.5 & 1.0 \\
\hline \multicolumn{4}{c}{ Planar probe } \\
$T_{e}$ (single $T$ fit) & 2130 & 3694 & \multicolumn{1}{c}{5652} \\
STD (single $T$ fit) & $5(-2)$ & $7.0(-2)$ & $5.0(-2)$ \\
STD (two $T$ fit) & $8.0(-6)$ & $7.7(-6)$ & $9.3(-5)$ \\
\multicolumn{5}{c}{} \\
$T_{e}$ (single $T$ fit) & Cylindrical probe \\
STD (single $T$ fit) & 2133 & 3698 \\
STD (two $T$ fit) & $5.4(-2)$ & $7.2(-2)$ & 5655 \\
\multicolumn{5}{c}{$5.4(-2)$} \\
$T_{e}$ (single $T$ fit) & $8.0(-6)$ & $7.7(-6)$ & $7.2(-6)$ \\
STD (single $T$ fit) & Spherical probe & \\
STD (two $T$ fit) & $5.4(-2)$ & 7678 & 5633 \\
\hline \hline
\end{tabular}

efficients. A Newtonian scheme is used to refine the fit to obtain a temperature that minimizes the standard deviation, STD.

A similar method was used to fit the curves for two temperatures using the formula:

$I=a+b V+c \exp \left(\frac{11604.45 V}{T_{1}}\right)+d \exp \left(\frac{11604.45 V}{T_{2}}\right)$.

The ratio of coefficients $d$ and $c$ determine the ratio of the densities of the high and low temperature components. The domains of the two temperatures are kept distinct to ensure the uniqueness of the fit parameters. In a flight application, where perhaps millions of curves may have to be fitted, a faster scheme would be devised. The present fit procedure, however, is adequate to demonstrate the nature of the twotemperature fitting procedure.

These fit procedures were applied to the three cases of different probe geometry, planar, cylindrical, and spherical, and to three distribution functions, purely Maxwellian, twotemperature Maxwellian and a Maxwellian with a superimposed electron beam. When the two-temperature fits were applied to the one-temperature curves, the density derived for the second component was essentially zero, thus confirming that only a single temperature was present. The fits to the single Maxwellian are not shown in the table because they reproduce the input values of $T_{e}$ and $N_{e}$ with greater than $1 \%$ accuracy corresponding to STDs of less than $5 \times 10^{-5}$.
Table I lists the derived temperatures and fit STDs for a two-temperature distribution and for different density ratios of the two components. The numbers in parenthesis are power of 10 , e.g., $3.2(-5)=3.2 \times 10^{-5}$. The curve to be fitted were obtained using the appropriate equations that were presented earlier. We assumed no digitization errors and used 226 voltage values between -3 and $0 \mathrm{~V}$. The theoretical temperatures were 1000 and $10000 \mathrm{~K}$, respectively. Although not shown in Table I, the two-temperature fits retrieved the two temperatures and three density ratios with negligible error. The ratio of fit parameters, $d /(c+d)$ for the two-temperature least-squares fit were, $0.44,0.61$, and 0.76 which agree with the theoretical values corresponding to density ratios $n_{2} / n_{1}$ of $0.25,0.5$, and 1.0 , respectively. These ratios are related by the formula,

$$
\frac{d}{d+c}=\frac{f}{1+f}
$$

where

$$
f=\frac{n_{2}}{n_{1}} \sqrt{\frac{T_{2}}{T_{1}}} .
$$

Note that the STDs of the single-temperature fits are much higher than those obtained when the more appropriate two-temperature fits are performed. All three geometries provide very low STDs for the two-temperature fits, indicating that all are appropriate for measuring two-temperature electron populations. Table I also demonstrates the vast improvement in the STD when a two-temperature fit is used instead of the simple single-temperature fit. We find that a twotemperature fit still works when the original distribution contains only one-temperature component. The STD does not improve significantly relative to a single-temperature fit. This result suggests that the presence of a two-temperature plasma can be confirmed by comparing the STDs for a twotemperature fit and a single-temperature fit. A singletemperature plasma is present if the STDs are low for both types of fit. A two-temperature plasma is present if the single-temperature fit yields a high STD and a twotemperature fit yields two temperatures, both with small STDs. If neither of these outcomes occur, then a more complicated energy distribution must be present.

TABLE II. $T_{e}$ and STD from single- and two-temperature fits curve produced by a Maxwellian component at $1000 \mathrm{~K}$ and a $1 \mathrm{eV}$ beam with a superposed $2000 \mathrm{~K}$ temperature.

\begin{tabular}{lccc}
\hline \hline \multicolumn{1}{c}{$n_{2} / n_{1}$} & 0.25 & 0.5 & 1.0 \\
\hline & \multicolumn{2}{c}{ Cylindrical probe } & \\
$T /$ STD (single $T$ fit) & $4935 / 0.14$ & $11,426 / 9.8(-2)$ & $22,260 / 5.0(-2)$ \\
& Two-temperature fit yields $T_{1}=1000 \mathrm{~K}, T_{2}=36800 \mathrm{~K}$ & $3.0(-2)$ \\
STD (two $T$ fit) & $4.8(-3)$ & $1.3(-2)$ & 0.98 \\
$d /(c+d)$ (two $T$ fit) & 0.865 & 0.94 & \\
& Spherical probe & & \\
$T /$ STD (single $T$ fit) & $3100 / 8.8(-2)$ & $5726 / 8.0(-2)$ & $6483 / 4.0(-2)$ \\
& Two-temperature fit yields $T_{1}=1000 \mathrm{~K}, T_{2}=12000 \mathrm{~K}$ & $6.7(-3)$ \\
STD (two $T$ fit) & $1.1(-3)$ & $3.0(-3)$ & 0.87 \\
$d /(c+d)$ (two $T$ fit) & 0.57 & 0.75 & \\
\hline \hline
\end{tabular}


Next, it is shown what happens when one of these more complicated energy distributions is present. Table II shows the $T_{e}$ and STD results obtained when single- and twotemperature fits are performed on the curves shown in Figs. 3(a)-3(c). These curves were derived for the case where both a directed electron beam and a single-temperature plasma are present. The thermal plasma has a temperature of $1000 \mathrm{~K}$, while the beam was given a directed energy of $1 \mathrm{eV}$ and a superposed temperature of $2000 \mathrm{~K}$. The family of density ratios are as shown in the figures.

Since the curves do not appear as simple exponentials, it is not surprising that the STDs are high for both single- and two-temperature fits. The fit data for the planar probe are not shown because the STDs are so large as to make the results meaningless.

\section{DISCUSSION}

While this work has presented and illustrated the Langmuir probe theory for non-Maxwellian electron populations, we have also tried to convey some sense of how one might proceed to use these results in the analysis of experimental $I-V$ curves obtained in space. In our experience, the vast majority of such $I-V$ curves can be fitted very well using the original Langmuir probe theory for a single-temperature and density plasma. Under certain conditions, however, the effects of photoelectrons or impact secondaries can be seen in the $I-V$ curves as a high energy tail in the retarding regions. ${ }^{4}$ Although we have not experienced it in the ionosphere, one can be sure that a Langmuir probe would be sensitive to the presence of an energetic electron beam, and/or any secondaries that it might produce.

The presence of secondaries is not usually a surprise to the experimenter who understands how spacecraft interact with the atmosphere and knows what other devices onboard may produce interfering plasma populations. Therefore, one starts off with a good idea of which kinds of distributions to employ in fitting such curves. In our experience, most such curves can be fitted by introducing into the fit a second Maxwellian component. If the spacecraft is highly positive, or if an electron beam source is known to be onboard, it may be necessary to add or substitute an electron beam term into the fitting routine. In principle, some such combination of Maxwellian and beam sources can reproduce almost any $I-V$ curve that is likely to be encountered. However, the parameters derived from the $I-V$ curves may not always give either the original ionosphere component or the secondary components. Interactions among the various populations could conceivably produce a final distribution at the probe location that is not a simple superposition of the components that produced it. A detailed evaluation of this possibility would be required to validate that assumption in each case.

We showed that all three probe geometries are not equally suitable for the measurement of $T_{e}$. An earlier work by Hoegy ${ }^{16}$ showed how different measurement techniques, for example, radar backscatter, ac mode Langmuir plate, etc. using distinct methods of deriving temperature tend to give different temperature values when the distribution is nonMaxwellian. Thus distinct techniques or instrument type also lead to different plasma probe results. The figures demonstrated that electron saturation regions differ greatly with collector geometry. The saturation region of the cylindrical probe is nearly independent of $T_{e}$, whereas this region is highly temperature dependent for the planar and spherical probes. We showed that the retarding region of the $I-V$ curves is identical for all three probe geometries when the distribution is isotropic. The first derivative of the retarding regions gives the $1 \mathrm{D} \mathrm{VDF}$, while the second derivative yields the full 3D VDF. When the energy distribution is anisotropic, it is not always possible to derive the distribution uniquely, but some information can be obtained from the derivatives. For example, the first derivative of the planar probe current and the second derivative of the spherical probe current give averages of the anisotropic distribution functions.

${ }^{1}$ R. L. F. Boyd and N. D. Twiddy, Proc. R. Soc. London, Ser. A 250, 53 (1959).

${ }^{2}$ L. H. Brace, Measurement Techniques in Space Plasmas: Particles, edited by R. F. Pfaff, J. E. Borovsky, and D. F. Young (Am. Geophys. Union, Washington, DC, 1998)

${ }^{3}$ E. C. Whipple, L. H. Brace, and L. W. Parker, Proceedings of the 17 th ESLAB Symposium on Spacecraft Interactions, ESA Report SP-198, 13-16 September 1983, p. 127.

${ }^{4}$ L. H. Brace, R. F. Theis, H. G. Mayr, S. A. Curtis, and J. G. Luhmann, J. Geophys. Res. 87, 199 (1982).

${ }^{5}$ W. C. Knudsen and K. L. Miller, J. Geophys. Res. 90, 2695 (1985).

${ }^{6}$ J. M. Mott-Smith and I. Langmuir, Phys. Rev. 28, 727 (1926).

${ }^{7}$ P. J. Bowen, R. L. F. Boyd, D. L. Henderson, and A. P. Willmore, Proc. R. Soc. London, Ser. A 250, 53 (1959).

${ }^{8}$ G. L. Wrenn, Proc. IEEE 57, 1072 (1969).

${ }^{9}$ W. R. Hoegy and L. E. Wharton, J. Appl. Phys. 44, 5365 (1973).

${ }^{10}$ D. H. Clark, W. J. Raitt, and A. P. Willmore, J. Atmos. Terr. Phys. 35, 63 (1973).

${ }^{11}$ R. Schunk and D. Watkins, J. Geophys. Res. 86, 91 (1981).

${ }^{12}$ V. L. Federev, Sov. Phys. Tech. Phys. 30, 554 (1985).

${ }^{13}$ E. D. Rainville, Special Functions (MacMillan, New York, 1960).

${ }^{14}$ W. H. Press, S. A. Teukolsky, W. T. Velterling, and B. P. Flannery, Numerical Recipes in FORTRAN (Cambridge University Press, Cambridge, 1992).

${ }^{15}$ J. P. Krehbiel, L. H. Brace, R. F. Theis, J. R. Cutler, W. H. Pinkus, and R. B. Kaplan, IEEE Trans. Geosci. Remote Sens. GE-18, 49 (1980).

${ }^{16}$ W. R. Hoegy, J. Geophys. Res. 76, 8333 (1971). 